NASA Contractor Report 3271

\title{
Numerical Simulation of Shear Flows Over Wavy Boundaries
}

Steven A. Orszag

CONTRACT NAS1-15383

APRIL 1980 
NASA Contractor Report 3271

\section{Numerical Simulation of Shear Flows Over Wavy Boundaries}

Steven A. Orszag

Cambridge Hydrodynamics, Inc.

Cambridge, Massachusetts

Prepared for

Langley Research Center

under Contract NAS1-15383

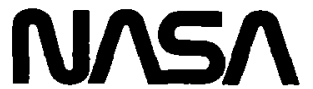

National Aeronautics

and Space Administration

Scientific and Technical

Information Office

1980 
a Amplitude of wall displacement

A Fourier-Chebyshev amplitude of $\mathrm{v}$; see (16)

$\mathrm{C}_{\mathrm{p}}$ Pressure coefficient

f Wall shape function

$\mathrm{G}_{\mathrm{k}}$ See (20)

$\mathrm{H} \quad \mathrm{H}(\mathrm{Y})=\mathrm{dY} / \mathrm{dn} ;$ see (17)

$\mathrm{k}$ Wavenumber of wall displacement

$\overline{\mathrm{k}} \quad$ Turbulent kinetic energy

m X-wavenumber

$\mathrm{N}$ Number of retained modes in $\mathrm{x}_{1}$ or $\zeta$

$\mathrm{p}$ Pressure

$\operatorname{Re}_{\mathrm{x}} \mathrm{Ux} / \mathrm{\nu}$, Reynolds number

$t$ Time

$T$ Reynolds stress

$T_{n}$ Chebyshev polynomial of degree $n$

$u_{k} \quad$ Eigenfunction; see (18)

$\mathrm{u}$ Velocity in $\mathrm{x}$-direction

$\mathrm{U}$ Free-stream velocity in $\mathrm{x}$-direction

$v \quad$ velocity in $y$-direction

$v_{k} \quad$ See (19)

$\mathrm{x}$ Unmapped Cartesian coordinate

$\mathrm{x}_{1} \quad \mathrm{x}_{1}=\mathrm{x}$

$\mathrm{x}_{2} \quad \mathrm{x}_{2}=\mathrm{y}$

$\mathrm{X}$ Mapped coordinate; see (14) or (15)

y Unmapped Cartesian coordinate

Y Mapped coordinate; see (14) or (15)

$\alpha \quad=1,2 ;$ coordinate label

$\Delta t \quad$ Time step

$\Delta \mathbf{x} \quad$ space discretization interval

$\Delta u \quad$ Velocity fluctuation

$\delta \quad$ Boundary layer thickness

$n$ Coordinate in conformal mapped system

$\phi_{0} \quad$ Phase of wall displacement 
$\lambda_{\mathrm{k}}$ Eigenvalue; see (18)

$\mu_{\mathrm{T}} \quad$ Eddy viscosity coefficient

$\checkmark \quad$ Viscosity

$\pi \quad \mathrm{Pi}$

$\rho \quad$ Density

$\bar{\rho} \quad$ Parameter used in (17)

$\sigma \quad$ Parameter used in viscous stabilization; see (13)

$\zeta$ Coordinate in conformally mapped system

Superscripts

- Convective update

^ Pressure update

- Viscous update

$=$ Viscous stabilization

- Derivative with respect to $\zeta+i n$; Also, fluctuation from mean flow 


\section{INTRODUCTION}

The study of mechanisms of possible drag reduction effects in flows over wavy walls as well as the study of mechanisms of generation of surface waves by wind requires understanding of the detailed flows over wavy surfaces. In this paper, we describe a computer code based on spectral methods to study two-dimensional incompressible flows in wavy geometries.

Work on flows in wavy geometries began with the classical analysis of Kelvin and Helmholtz of linearized inviscid surface waves ${ }^{1}$. In the absence of mean-flow-shear effects, inviscid theory predicts that, for wall displacements of the form $y=a \cos \left(k x+\phi_{0}\right)$ with $\mathrm{ka} \ll 1$ (so that the problem may be linearized in terms of $\mathrm{ka}$ ), the pressure distribution is $180^{\circ}$ out of phase with the wall displacement and the pressure coefficient $c_{p}=\left|p_{2}\right|_{\max } / \frac{1}{2} \rho U^{2}$ has the value $2 \mathrm{ka}$ at the wall. Miles ${ }^{2}$ extended the inviscid theory of account for shear in the mean flow, still restricting attention to very gentle waves. Miles' theory improves the prediction of the pressure coefficients. However, because of the inviscid nature of the analysis, the pressure phase shift at the wall is still $180^{\circ}$. 
Benjamin ${ }^{3}$ analyzed high Reynolds number laminar flows over wavy walls including the effects of shear still assuming ka $<1$. Benjamin's theory typically predicts a pressure phase shift of $210^{\circ}$ relative to the displacement of a solid wavy houndary. The theory of Miles and Benjamin has also been extended to turbulent flows. Davis ${ }^{4}$ analyzes turbulent flow over wavy surfaces by postulating that Reynolds stresses are, to a first approximation, constant along lines of constant $n$, where $(\zeta, \eta)$ is a first-order accurate orthogonal coordinate system with the wavy boundary at $\eta=0$. Davis' analysis is, therefore, also limited to small ka. Markatos ${ }^{5}$ has recently presented numerical results for transfer of heat, mass and momentum in flow over an evaporating wavy water surface. While Markatos' numerical results are at best firstorder accurate in space, they go a long way towards providing understanding of the nature of flow over wavy walls.

The work described in this paper extends previous work in several ways. The full time-dependent two-dimensional Navier-stokes equations are solved using spectral methods to achieve high spatial accuracy and high-order time-splitting methods together with conformal mapping methods to allow simulation of flow over steep waves. Results for laminar flows at 
Reynolds numbers up to $\operatorname{Re}_{x}=4 \times 10^{5}$ are presented in Sec. III. Turbulent flows over wavy walls are simulated by using a spatially varying eddy viscosity distribution. Some preliminary results and comparisons with experiment are given in Sec. IV.

\section{METHODS OF SOLUTION}

The two-dimensional Navier-stokes equations

are

$$
\frac{\partial v_{\alpha}}{\partial t}+\sum_{\beta=1}^{2} v_{\beta} \frac{\partial v_{\alpha}}{\partial x_{\beta}}=-\frac{\partial p}{\partial x_{\alpha}}+\sum_{\beta=1}^{2} \frac{\partial}{\partial x_{\beta}} T_{\alpha \beta}
$$

with $\alpha=1,2$,

$$
\sum_{\alpha=1}^{2} \frac{\partial \mathrm{v}_{\alpha}}{\partial \mathrm{x}_{\alpha}}=0
$$

where the stress tensor may include both laminar

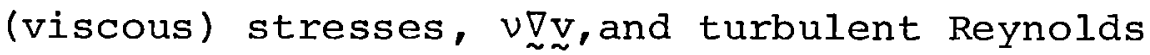
stresses (to be discussed briefly in sec. IV below). Eqs. (1) - (2) are to be solved in the region

$$
0 \leq x_{1}<2 \pi, f\left(x_{1}, t\right) \leq x_{2}<\infty
$$

above the wall $x_{2}=f\left(x_{1}, t\right)$. In the present paper, we restrict attention to surfaces of the form

$$
f\left(x_{1}, t\right)=a \cos k x
$$

independent of $t$ with periodic inflow-outflow boundary conditions in $x_{I}$, viz. 


$$
v_{\alpha}\left(x_{1}+2 \pi, x_{2}, t\right)=v_{\alpha}\left(x_{1}, x_{2}, t\right)
$$

Extensions to study the effects of time-dependent geometries and inflow-outflow boundary conditions are presently being made.

A conformal mapping technique ${ }^{6}$ is used to transform the region (4) into the region

$$
0 \leq \zeta \leq 2 \pi, 0 \leq n<\infty
$$

If $a \leqslant 1$, the conformal mapping coefficients are accurately generated in only order $\mathrm{N}$ log $\mathrm{N}$ operations where $\mathrm{N}$ is the resolution along $\mathrm{x}_{1}(\zeta)$, so time dependent geometries can be efficiently handled. A further (non-conformal) stretching transformation of $\eta$ is used to implement the spectral methods described below see Eqs. (14)-(15)

Eqs. (1) - (2) are solved numerically by a fractional step procedure. The fractional steps are: (i) Convective update .

An intermediate field $v_{\alpha}$ is found so that

$$
\tilde{\mathrm{v}}_{\alpha}=\mathrm{v}_{\alpha}(t)+\frac{3}{2} \Delta t \mathrm{~N}_{\alpha}(t)-\frac{1}{2} \mathrm{~N}_{\alpha}(t-\Delta t) \Delta t
$$

where

$$
\mathrm{N}_{\alpha}=\sum \mathrm{v}_{\beta} \frac{\partial}{\partial \mathrm{v}_{\beta}} \mathrm{v}_{\alpha}
$$

Here a second-order accurate Adams-Bashforth scheme is applied. 


\section{(ii) Pressure update}

$$
\begin{aligned}
& \hat{v}_{\alpha}=\tilde{v}_{\alpha}-\frac{\partial \zeta}{\partial x_{\alpha}} \frac{\partial p}{\partial \zeta}-\frac{\partial \eta}{\partial x_{\alpha}} \frac{\partial p}{\partial \eta} \\
& \sum_{\alpha=1}^{2}\left(\frac{\partial \zeta}{\partial x_{\alpha}} \frac{\partial \hat{v}_{\alpha}}{\partial \zeta}+\frac{\partial \eta}{\partial x_{\alpha}} \frac{\partial \hat{v}_{\alpha}}{\partial \eta}\right)=0
\end{aligned}
$$

with the boundary condition

$$
\sum_{\alpha=1}^{2} \frac{\partial \eta}{\partial x_{\alpha}} \hat{v}_{\alpha}=0 \text { at } \eta=0
$$

Substituting (7) into (8) gives the Poisson equation

$$
\frac{\partial^{2} p}{\partial \zeta^{2}}+\frac{\partial^{2} p}{\partial \eta^{2}}=\frac{1}{\left|F^{\prime}\right|^{2}} \sum_{\alpha=1}^{2}\left(\frac{\partial \zeta}{\partial x_{\alpha}} \frac{\partial \tilde{v}_{\alpha}}{\partial \zeta}+\frac{\partial \eta}{\partial x_{\alpha}} \frac{\partial \tilde{v}_{\alpha}}{\partial \eta}\right)
$$

with

$$
\left|F^{\prime}\right|^{2}=\sum_{\alpha=1}^{2}\left(\frac{\partial \zeta}{\partial x_{\alpha}}\right)^{2}=\sum_{\alpha=1}^{2}\left(\frac{\partial \eta}{\partial x_{\alpha}}\right)^{2},
$$

where the conformal nature of the map from $\left(x_{1}, x_{2}\right)$ to $(\zeta, n)$ is used. 
(iii) Viscous update

$$
\overline{\mathrm{v}}_{\alpha}=\hat{\mathrm{v}}_{\alpha}+\Delta t \sum_{\alpha=1}^{2} \frac{\partial}{\partial \mathrm{x}_{\beta}} \mathrm{T}_{\alpha \beta}(\mathrm{t})
$$

with the boundary conditions

$$
\overline{\mathrm{v}}_{\alpha}=0 \text { at } n=0
$$

This Euler step is only first-order accurate but will be improved upon later. The time step restrictions for numerical stability of (12) are particularly severe, especially in the case of turbulent flows, so the next fractional step is designed to stabilize the scheme.

$$
\begin{aligned}
& \text { (iv) } \frac{\text { Viscous }}{\text { stabilization }} \\
& \overline{\bar{v}}_{\alpha}-\frac{\sigma}{\left|F^{\prime}\right|_{\min }^{2}} \nabla_{\zeta}^{2} \bar{v}_{\alpha}=\bar{v}_{\alpha}-\frac{\sigma}{\left|F^{\prime}\right|_{\min }^{2}} \nabla_{\zeta}^{2} v_{\alpha}(t)
\end{aligned}
$$

with

$$
\overline{\bar{v}}_{\alpha}=0 \text { at } \eta=0 \text {, }
$$

where $\nabla_{\zeta}^{2}=\partial^{2} / \partial \zeta^{2}+\partial^{2} / \partial \eta^{2} \quad$ is the Laplacian with respect to $\zeta, \eta$. Here $\sigma$ is a parameter chosen to achieve optimal stabilization and accuracy.

(v) Local extrapolation

For time dependent problems requiring accurate calculation of transient effects, a local extrapolation procedure ${ }^{7}$ is used to achieve global second-order accuracy in time. Further details and caveats on the splitting procedure described above are given in Ref. 7 . 
In order to implement this algorithm in a spectral method ${ }^{8}$, the semi-infinite region $0 \leq \zeta \leq 2 \pi$, $0 \leq n<\infty$ is mapped into the finite rectangular domain

$$
0 \leq X \leq 2 \pi, \quad-1 \leq Y<1
$$

by

$$
X=\zeta, \quad Y=\frac{2 \eta}{n+L}-1
$$

or

$$
X=\zeta, \quad Y=1-2 e^{-n / L}
$$

for some suitable mapping scale $\mathrm{L}$.

Next, all dependent variables are expanded in Fourier-Chebyshev series of the form

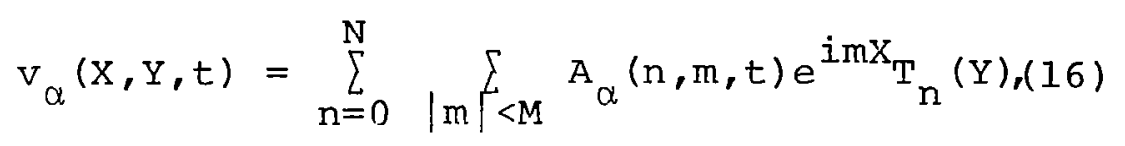

where $T_{n}(Y)=\cos \left(n \cos ^{-1} Y\right)$ is the Chebyshev polynomial of degree $n$.

Technical details regarding spectral methods and their implementation are given in Ref. 8 and references given therein. For illustration purposes, the method of solution of the Poisson equation (10) or (13) will now be described. After the transformation (14) or (15), the equation to be solved is of the form

$$
\bar{\rho} \mathrm{u}+\frac{\partial^{2} \mathrm{u}}{\partial \mathrm{X}^{2}}+\mathrm{H}(\mathrm{Y}) \frac{\partial}{\partial \mathrm{Y}} \mathrm{H}(\mathrm{Y}) \frac{\partial \mathrm{u}}{\partial \mathrm{Y}}=\mathrm{g}(\mathrm{X}, \mathrm{Y}) \text {. }
$$


Introducing the eigenvalue problem

$$
H(Y) \frac{\partial}{\partial Y} H(Y) \frac{\partial u_{k}}{\partial Y}=\lambda_{k} u_{k}
$$

subject to the appropriate homogeneous boundary conditions, say $u_{k}=0$, it follows that

$$
\begin{aligned}
& u(X, Y)=\sum_{k} v_{k}(X) u_{k}(Y) \\
& g(X, Y)=\sum_{k} G_{k}(X) u_{k}(Y)
\end{aligned}
$$

where $v_{k}$ satisfies

$$
\bar{\rho} v_{k}+\frac{\partial^{2} v_{k}}{\partial x^{2}}+\lambda_{k} V_{k}=G_{k} \text {. }
$$

Then (21) is solved by Fourier transformation in $x$, so the solution $u$ to (10) or (13) can be found by further matrix operations.

In the next Section, results are reported from this code using up to 64 Fourier modes and Chebyshev polynomials in each of the $X$ and $Y$ directions. Typically, the fractional step method with local extrapolation and tensor stress evaluation requires about 6 ms per retained mode per time step on a CDC 6600 computer. Time steps are restricted for stability to be less than the convective stability limit $\Delta x / U$. 
Results from three laminar flow runs will be described here. The first case is flow over a very gentle wave a cos $\mathrm{kx}$ with $\mathrm{k}=4 \pi \mathrm{cm}^{-1}$, $\mathrm{ka}=\pi / 1000$ at a Reynolds number $\operatorname{Re}_{\mathrm{x}}=4 \times 10^{5}$ at $x=2.9 \mathrm{~cm}$, so the boundary thickness $\delta=6.5 \sqrt{\frac{v \mathrm{x}}{\mathrm{U}_{\infty}}}$ $=0.3 \mathrm{~cm}$. A plot of the mapped grid used in the calculations of this flow is given in Fig. 1 , where only the portion of the grid near the wall is plotted. Since the $x$ and $y$ scales are different, the conformal nature of the map is obscured in the plot.

In Fig. 2 we plot contours of the pressure at $t=90$ (in nondimensional units in which $U_{\infty}=1$ ) after the solution has converged to a steady state. The calculation was performed using 32 Fourier modes in $X$ and 33 Chebyshev polynomials in $Y$. The contouring is done in the unmapped $x_{1}=x$ and $\mathrm{x}_{2}=\mathrm{y}$ coordinates. The phase of the pressure distribution lags $214^{\circ} \pm 5^{\circ}$ behind the surface displacement a cos $\mathrm{x}$ and the pressure coefficient $\begin{aligned} \mathrm{C}_{\mathrm{p}}=|\mathrm{p}|_{\max ^{/ \frac{1}{2} \rho} \mathrm{u}_{\infty}^{2}} & \text { is } \\ & \mathrm{C}_{\mathrm{p}} \simeq 1.76 \times 10^{-4}\end{aligned}$

at the wall. As mentioned above, the Kelvin-Helmholtz inviscid analysis predicts $C_{p}=2 k a \simeq 6.3 \times 10^{-3}$. On the other hand, Benjamin's theory ${ }^{3}$ predicts a phase shift of about $217^{\circ}$ with $C_{p}$ in good agreement with our numerical simulations. 
In Figs. 3 and 4 we plot contours of the velocity in the $x_{2}$ direction in the unmapped $x_{1}=x$ and $x_{2}=y$ coordinates. Fig. 4 is a blown-up portion of Fig. 3 that highlights the structure of the velocity $v_{2}$ near the wall. Note that the scale of the boundary layer is such that $u=0.96 \mathrm{U}_{\infty}$ at ky $\simeq 2.5$. In Fig. 5 we plot contours of $u_{1}$ in the $x-y$ plane blown-up to highlight the wall region. In Figs. 6 and 7 , we plot contours of the spanwise vorticity at $t=90$. Again, Fig. 7 is a blown-up version of Fig. 6 .

The second run is similar to the first except that the amplitude of the surface displacement is increased to

$$
\mathrm{ka}=5.5 \times 10^{-2}
$$

In Fig. 8, a contour plot is given of the pressure distribution at a nondimensional time $t=90$. The phase of the pressure distribution now lags about $212^{\circ}$ behind the surface while the pressure coefficient is increased to about

$$
c_{p} \simeq 3.3 \times 10^{-3} \text {. }
$$

Benjamin's theory still predicts a phase shift of about $217^{\circ}$ with $C_{p}$ in good agreement with the value obtained by numerical simulation. In Figs. 9-1l, we give contour plots in the wall region for the velocity components $\mathrm{v}_{1}, \mathrm{v}_{2}$ and spanwise vorticity, respectively, in the second run. 
The third run involves the simulation of flow studied experimentally by Kachanov et al ${ }^{9}$. Here $k=2.5 \mathrm{~cm}^{-1}$ while

$$
\mathrm{ka}=(5.3 \pm 0.1) \times 10^{-2}
$$

The calculation was performed at a Reynolds number $\operatorname{Re}_{\mathrm{X}}=1.1 \times 10^{5}$ to match the experiment, while $\mathrm{U}_{\infty}=530 \mathrm{~cm} / \mathrm{s}$ so the boundary layer thickness $\delta=6.5 \sqrt{\frac{V X}{U_{\infty}}}$ at $\mathrm{x}=3.06 \mathrm{~cm}$ is $\delta=0.6 \mathrm{~cm}$. Because the wavelength of the wall displacement is much longer in this run than in the second run, it turns out that the accurate simulation of this case is somewhat more difficult. Pressure pertubations due to the wall die out over a distance of order the wall wave-length which is much larger than $\delta$ so the resolution requirements in $\mathrm{Y}$ are more severe than for the first two cases. The results reported below were obtained using 32 Fourier modes in $\mathrm{X}$ and 65 Chebyshev polynomials in $\mathrm{Y}$.

In Fiq. 12, we plot contours of the pressure field for the third run after a steady state has been achieved. In Figs. 13 and 14, contour plots of the spanwise vorticity and velocity $v_{1}$, respectively, near the wall are given. Kachanov et al 9 measured the distribution of $x$-velocity $v_{1}=u$ fluctuations at a height of about $\delta$ above the wall. They found a fluctuation amplitude $\Delta u$ of

$$
\frac{\Delta u_{\text {expt }}}{U_{\infty}}=0.005 \pm 0.002
$$


with a phase shift of $40^{\circ} \pm 5^{\circ}$ relative to the wall. The computational results give

$$
\frac{\Delta u_{\text {comp }}}{U_{\infty}}=0.004 \pm 0.0002
$$

with a phase shift of $22^{\circ}+5^{\circ}$. The magnitude of the fluctuations agree well between laboratory and computer experiment. The discrepancy in the phase shifts may be due to the sensitivity of this quantity to measurement error or due to the precise manner of measurement (in the computer experiment, the fluctuations of $u$ were measured at constant $Y$ while in the laboratory experiment the measurements seem to have been conducted at constant $y$ ).

\section{TURBULENT FLOW RESULTS}

In this section, we report a simulation of Kendall's experiment ${ }^{10}$ of turbulent flow over a wavy wall. An eddy viscosity model is used to simulate the effects of the turbulence. The turbulent Reynolds stresses are modelled as

$$
\begin{aligned}
& -\rho \overline{v_{1}^{\prime 2}}=2 \mu_{T} \partial v_{1} / \partial x_{1}-\frac{2}{3} \rho \bar{k} \\
& -\rho \overline{v_{1}^{\prime} v_{2}^{\prime}}=\mu_{T}\left(\partial v_{1} / \partial x_{2}+\partial v_{2} / \partial x_{1}\right) \\
& -\overline{v_{2}^{\prime 2}}=2 \mu_{T} \partial v_{2} / \partial x_{2}-\frac{2}{3} \rho \bar{k}
\end{aligned}
$$


where primes indicate turbulent fluctuations, overbars are averages, $\mu_{T}$ is a (spatially varying) eddy viscosity coefficient and $\vec{k}$ is the turbulent kinetic energy.

The eddy coefficient $\mu_{\mathrm{T}}$ is modelled by use of a turbulent boundary layer code ${ }^{11}$ that includes effects of wall curvature and pressure gradients within a one-equation eddy-viscosity model. ${ }^{12}$ The actual pressure measurements of the Kendall experiment were used in this boundary layer code. The turbulent kinetic energy $k$ is modelled on the basis of flat plate data according to

$$
-\overline{v_{1}^{\prime} v_{2}^{\prime}} / \bar{k} \simeq 0.15
$$

A contour plot of the resulting eddy viscosity coefficient $\mu_{T}$ is given in Fig. 15.

Kendall's experiment is simulated using a wall displacement a cos $(\mathrm{kx})$ with $\mathrm{a}=0.32 \mathrm{~cm}$ and $k=0.62 \mathrm{~cm}^{-1}$. The mean flow velocity in the free stream is $U=500 \mathrm{~cm} / \mathrm{s}$ while the molecular viscosity is $0.14 \mathrm{~cm}^{2} / \mathrm{s}$. The calculations are performed using 32 Fourier modes in $\mathrm{X}$ and 33 Chebyshev polynomials in $Y$. The flow field is initialized using the result obtained from the boundary layer code ${ }^{11}$ corrected to be incompressible.

In Fig. 16, we plot contours of the velocity field $v_{1}$ in the $x_{1}$-direction at $t=2 \mathrm{~s}$. In Fig. 17, spanwise vorticity contours at $t=2 \mathrm{~s}$ are shown. Finally, in Figs. 18 and 19, contour plots of $v_{1}$ and $v_{2}$, respectively, at $t=4 \mathrm{~s}$ are given. It can be observed 
from Fig. 18 that a small separation bubble is developing in the flow at $t=4 \mathrm{~s}$. Separation seems to begin near $t=3.2 \mathrm{~s}$. While no separation was observed in Kendall's experiment, it is known that the laboratory flow is close to separation. There are several possible reasons for the appearance of a separation bubble in the computation. First, the eddy viscosity distribution $\mu_{T}$ is fixed for all time by the turbulent boundary layer calculation. Since the mean-flow velocity has evolved significantly from the boundary layer approximation, it seems reasonable to expect that $\mu_{T}$ should change also. Second, the modelling of normal Reynolds stresses in (22)-(24) may be suspect. A possible remedy for both these problems would be integrating a multiequation turbulence model at the same time as the mean flow equations. Further work is now planned in this direction. Finally, while the numerical resolution of the flow seems adequate in the wall region, there does seem to be need for more resolution at large distances from the wall. For this reason, higher resolution calculations with modified mappings in the $y$ direction are now underway.

\section{CONCIJUSION}

A computer code to solve the full two-dimensional Navier-Stokes equations in an arbitrary wavy geometry has been developed. The code has been run on both laminar and turbulent flows over wavy walls. The results have been compared with both available theory and experiment. For laminar flow, good agreement is achieved. For turbulent flow, the agreement is less satisfactory and may be explained by the simplified turbulence modelling used in these calculations. 
REFERENCES

1) Lamb, H. Hydrodynamics, 6th Ed., Cambridge University Press, Chap. IX, 1932.

2) Miles, J.W., J. Fluid Mech., Vol. 3, 1957, pp. $185-204$.

3) Benjamin, T.B., J. Fluid Mech., Vol. 6, 1959, pp. 161-205.

4) Davis, R.E., J. Fluid Mech., Vol. 42, 1970, pp. 721-731.

5) Markatos, N.C.G., Computer Methods in Appl. Mech. and Eng., Vol. 14, 1978, pp. 323-376.

6) Meiron, D., Orszag, S.A., and Israeli, M., Applications of Numerical Conformal Mapping, Cambridge Hydrodynamics Report No. 31, 1980.

7) Deville, M. and Orszag, S.A., Splitting Methods for Incompressible Flow Problems, Cambridge Hydrodynamics Report No. 32, 1980.

8) Gottlieb, D. and Orszag, S.A., Numerical Analysis of Spectral Methods, NSF-CBMS Monograph No. 26 , Soc. Ind. and Appl. Math., Philadelphia, 1977.

9) Kachanov, Yu. S., Kozlov, V.V., Kotjolkin, Yu.D. Levchenko, V. Ya., and Rudnitsky, A.L., Acta Astro., Vol. 2, 1975, pp. 557-559.

10) Kendal1, J.M.,J. Fluid Mech., Vol. 41, 1970, pp. 259-281.

11) Hixon, B.A., Beckwith, I.E., and Bushne11, D.M., Computer Program for Compressible Laminar or Turbulent Non-Similar Boundary Layers, NASA TMX-2140, 1971. 
12) Balasubramanian, R. Cary, A.M., Jr., Bushnell; D.M. and Ash, R.L., Influence of Transverse Surface Waves on Turbulent Boundary Layers, in Proc. Fifth Biennial Conf. on Turbulence in Liquids, Univ. of Missouri-Rolla, 1977, pp. 63-82. 


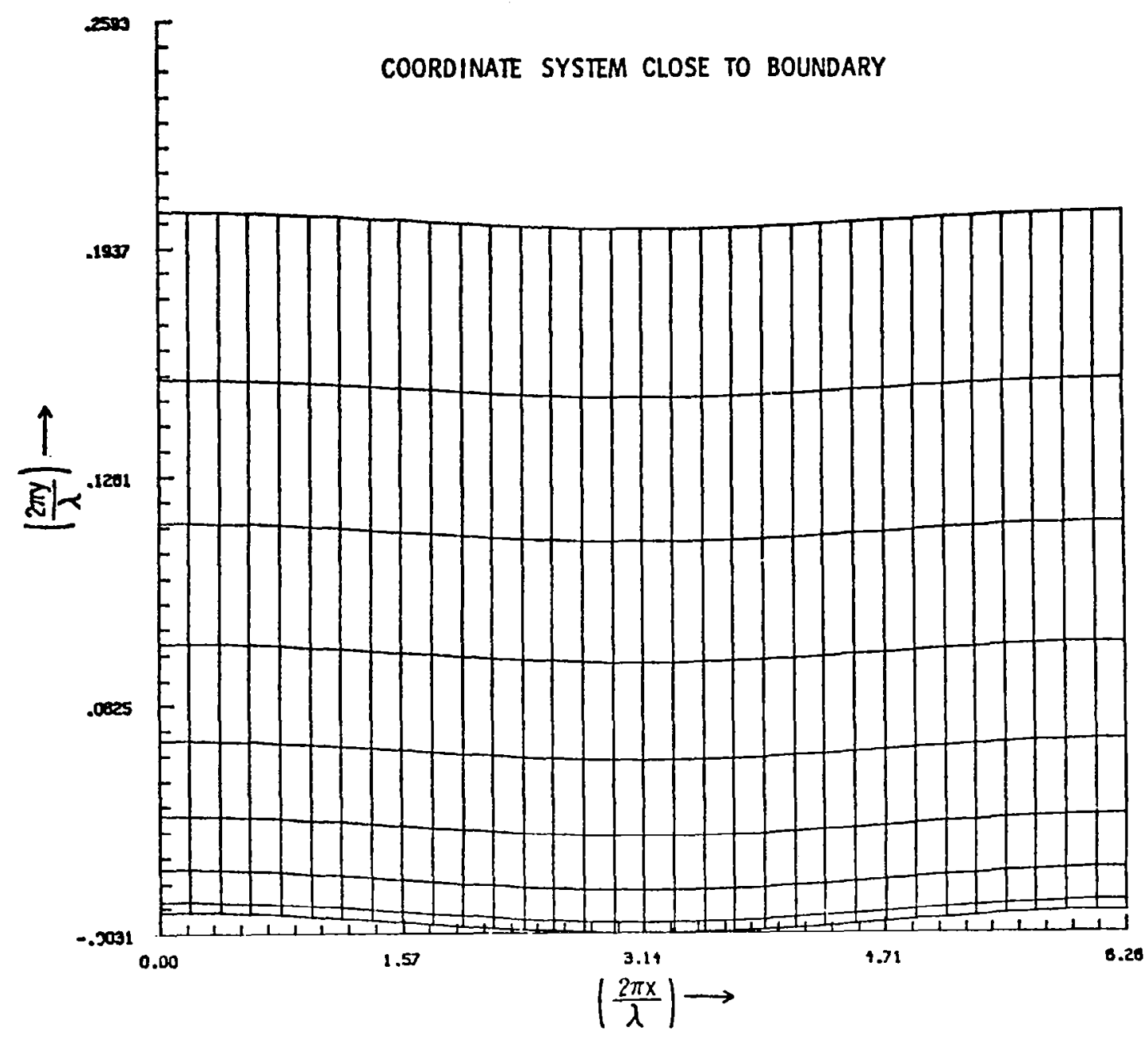

Figure 1. A plot of the coordinate system near the wall for Run 1. Here the wall displacement is $y=a \cos k x$ with $k=10 \pi$ and $a=10^{-4}$. 


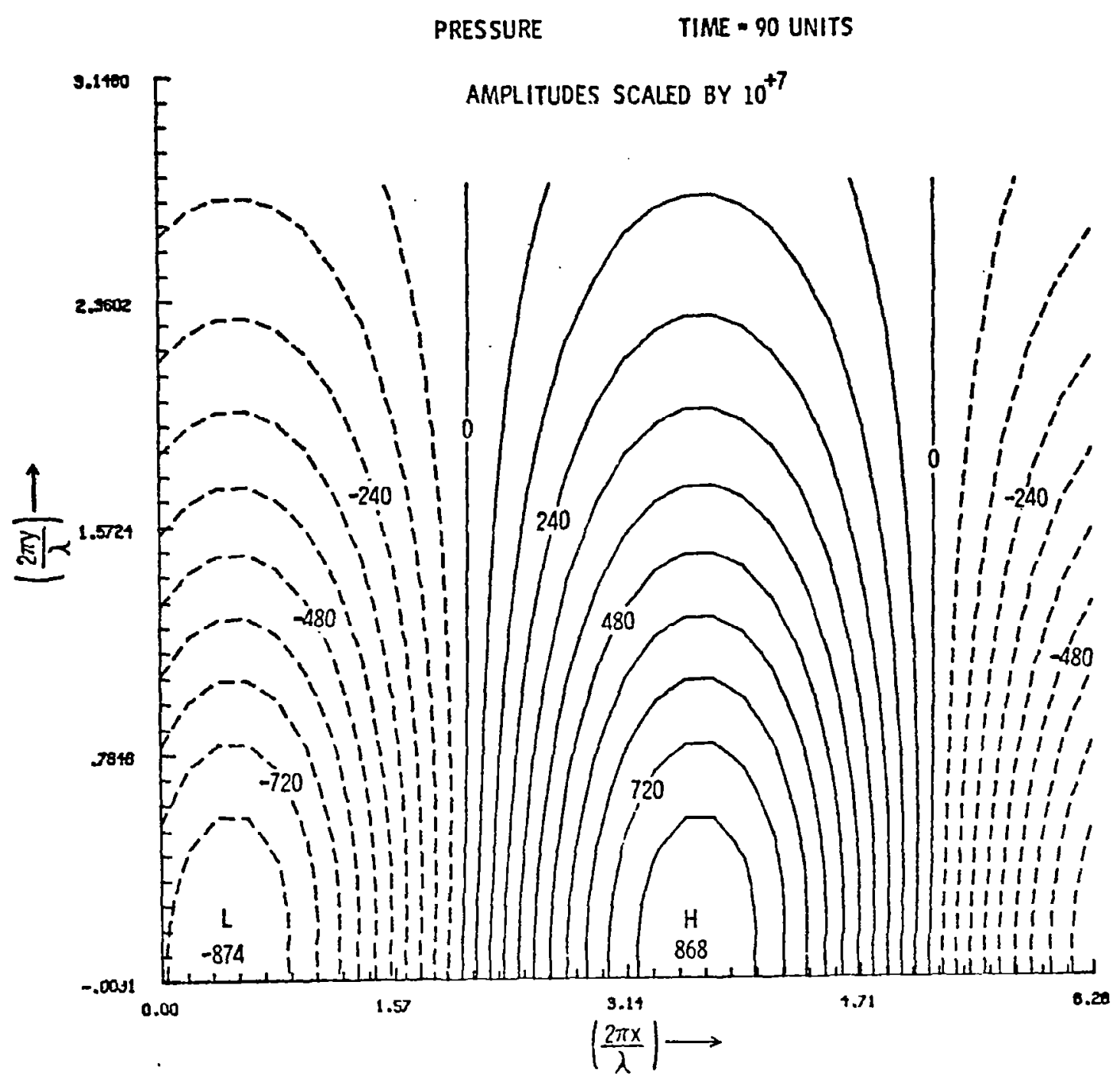

Figure 2. Contour plot of the pressure $p$ at $t=90$ for Run 1. 


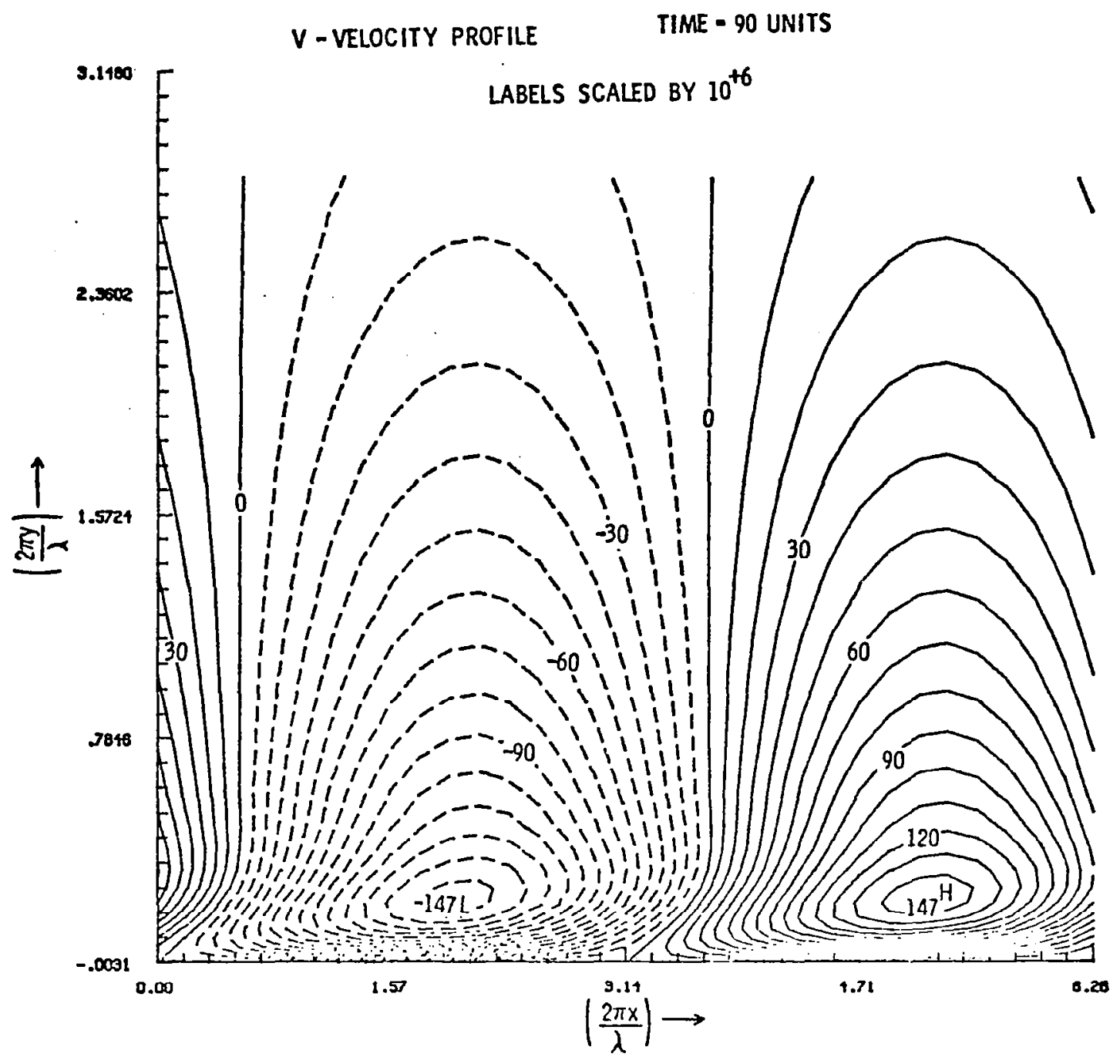

Figure 3. Contour plot of the $x_{2}$-velocity $v_{2}$ at $t=90$ for Run 1 . 


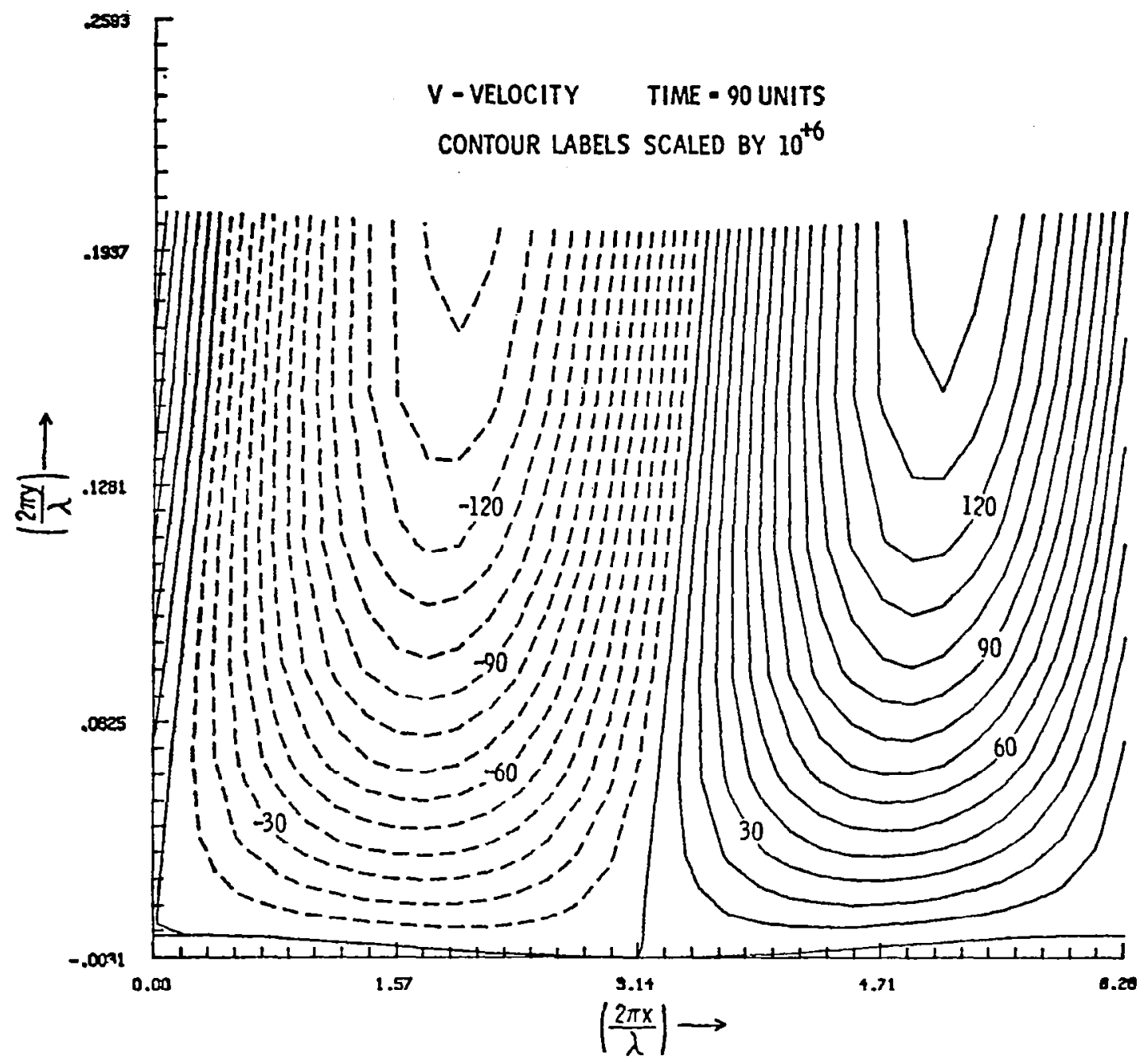

Figure 4. Same as Figure 3 except that the region near the wall is expanded. 


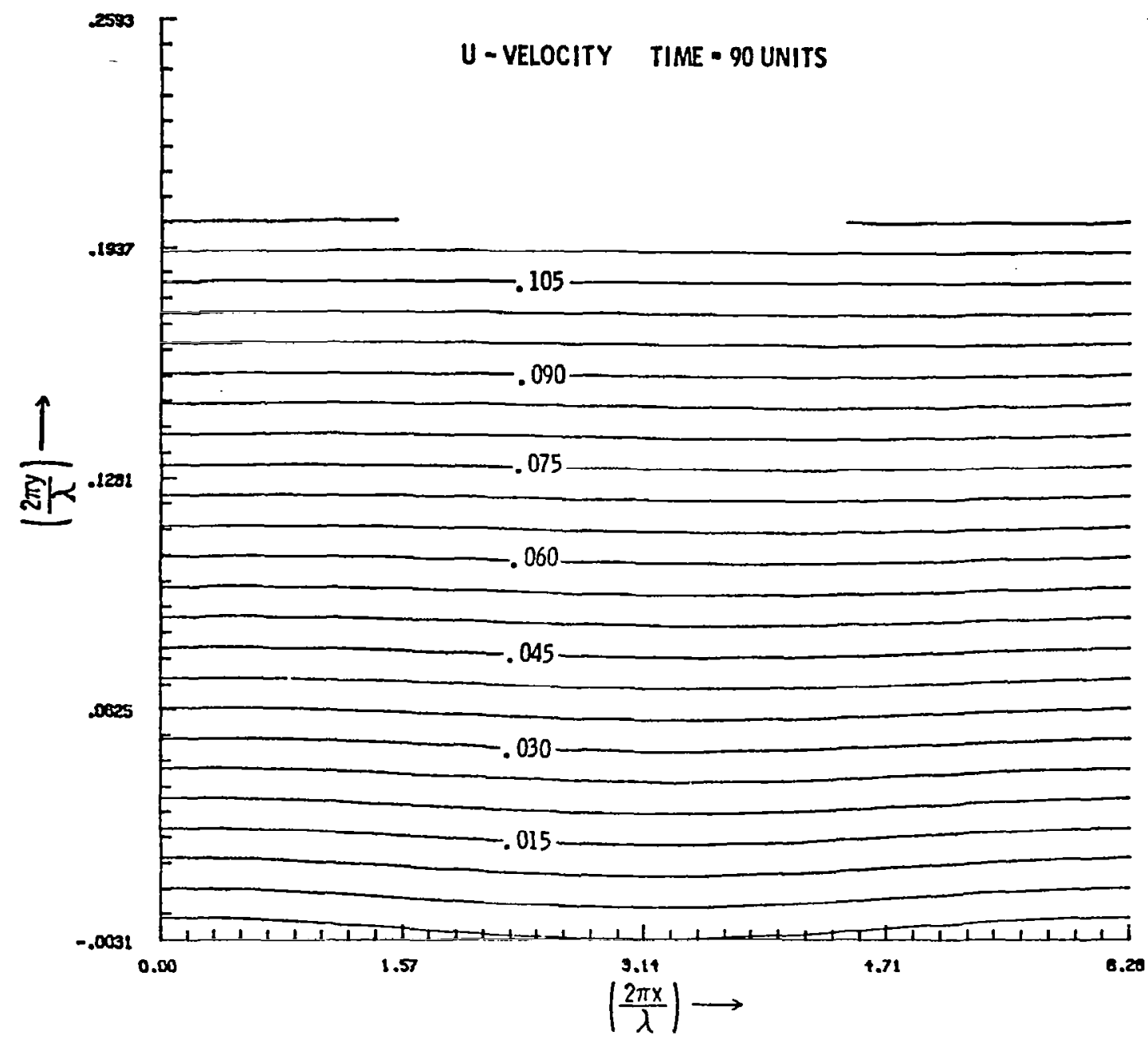

Figure 5. Contour plot of the $x_{1}$-velocity $v_{1}$ at $t=90$ near the wall for Run 1 . 


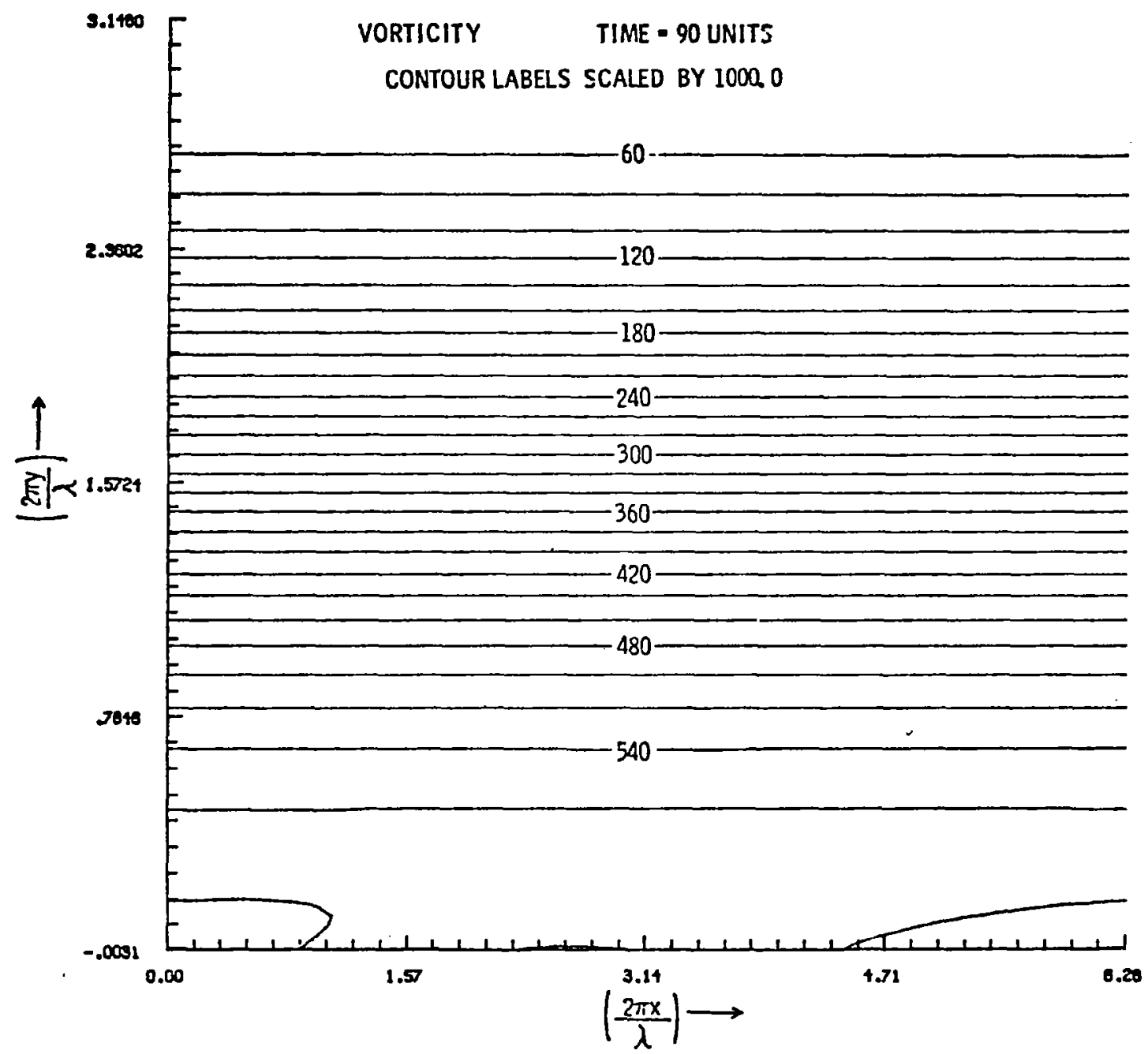

Figure 6. Contour plot of the spanwise vorticity in Run $I$ at $t=90$. 


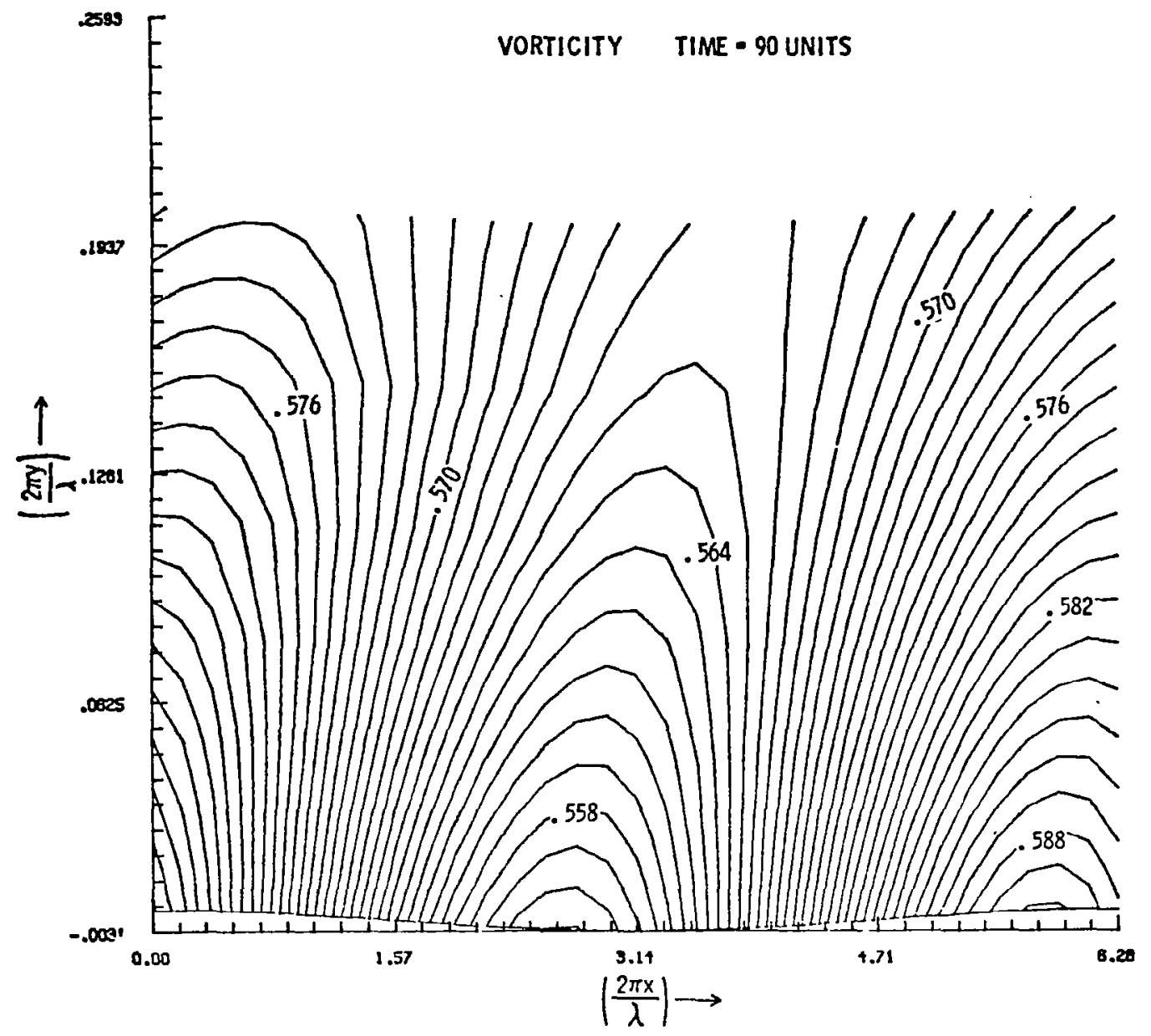

Figure 7. Same as Figure 6 except that the region near the wall is expanded. 


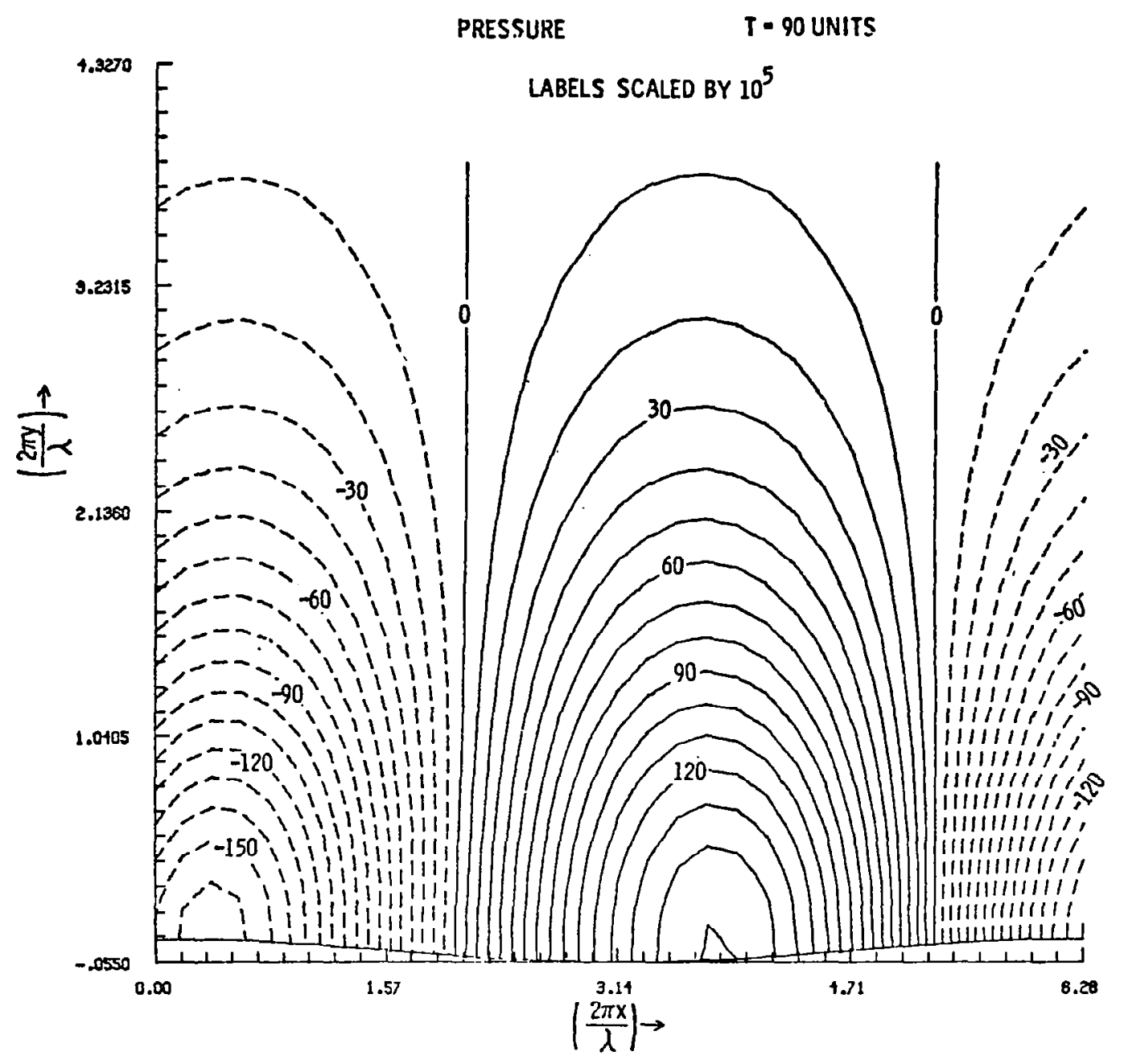

Figure 8. Contour plot of the pressure $p$ at $t=90$ in Run 2 . 


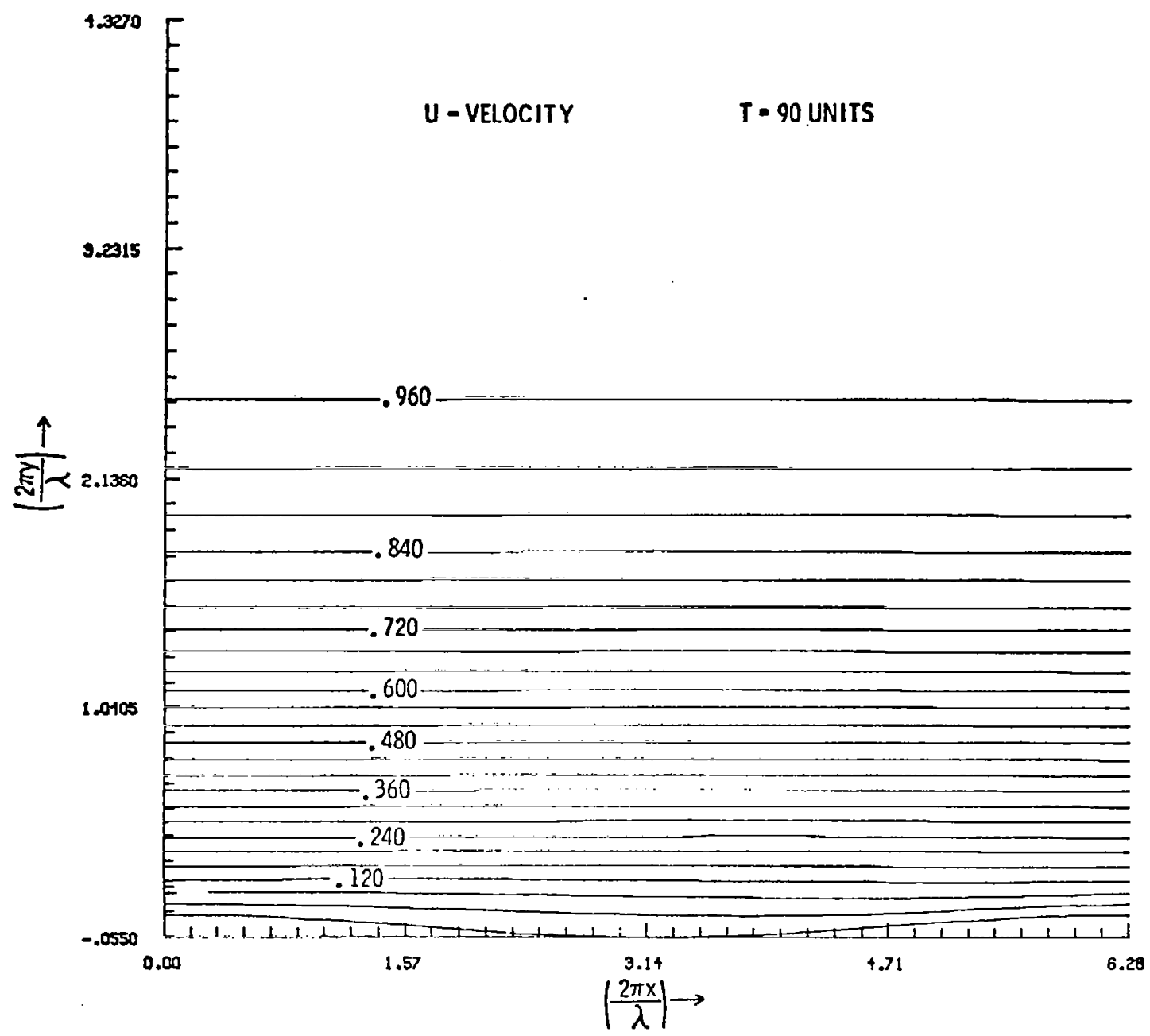

Figure 9. Contour plot of $v_{1}$ at $t=90$ in Run 2 . 


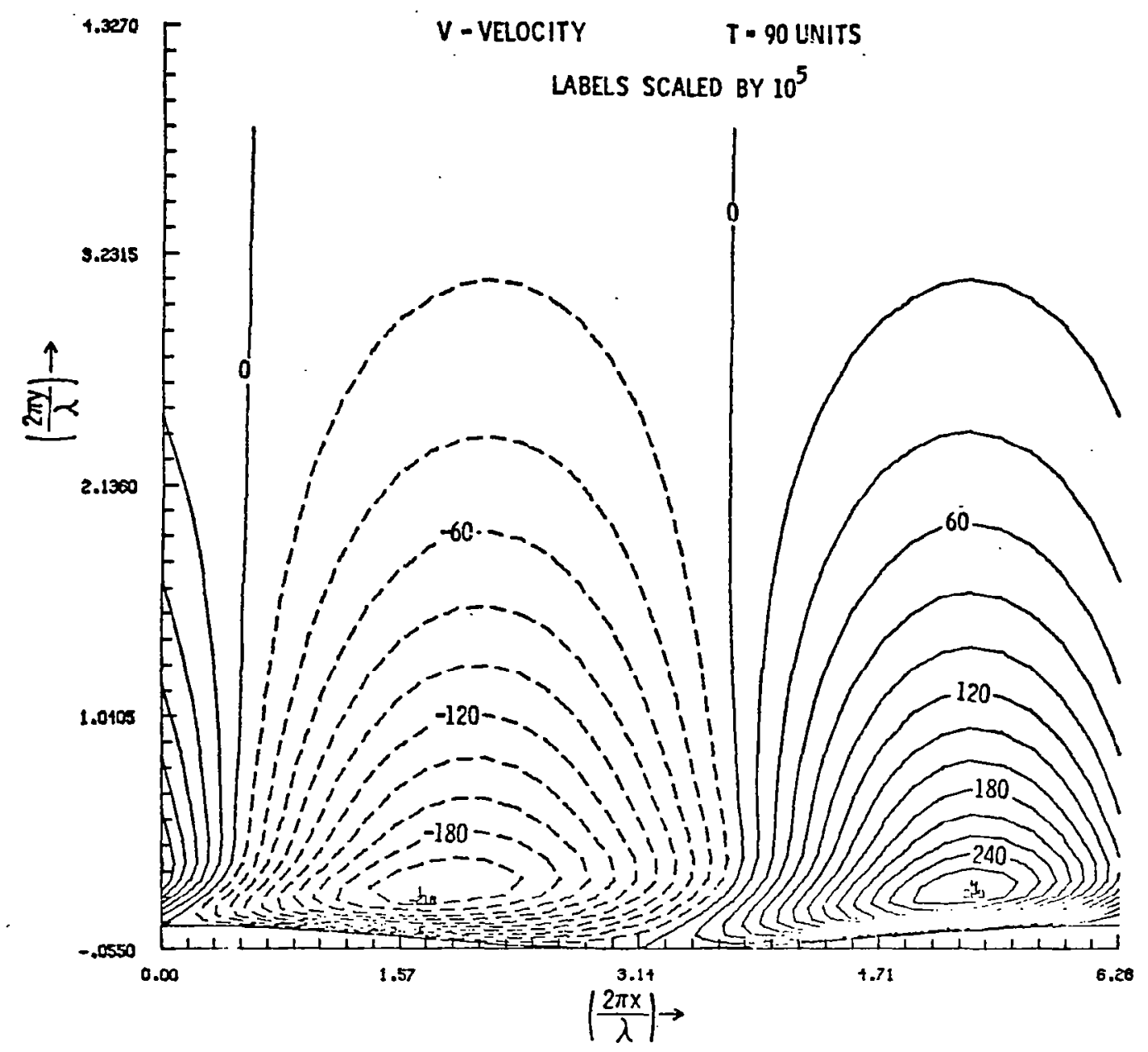

Figure 10. Contour plot of $v_{2}$ at $t=90$ in Run 2 . 


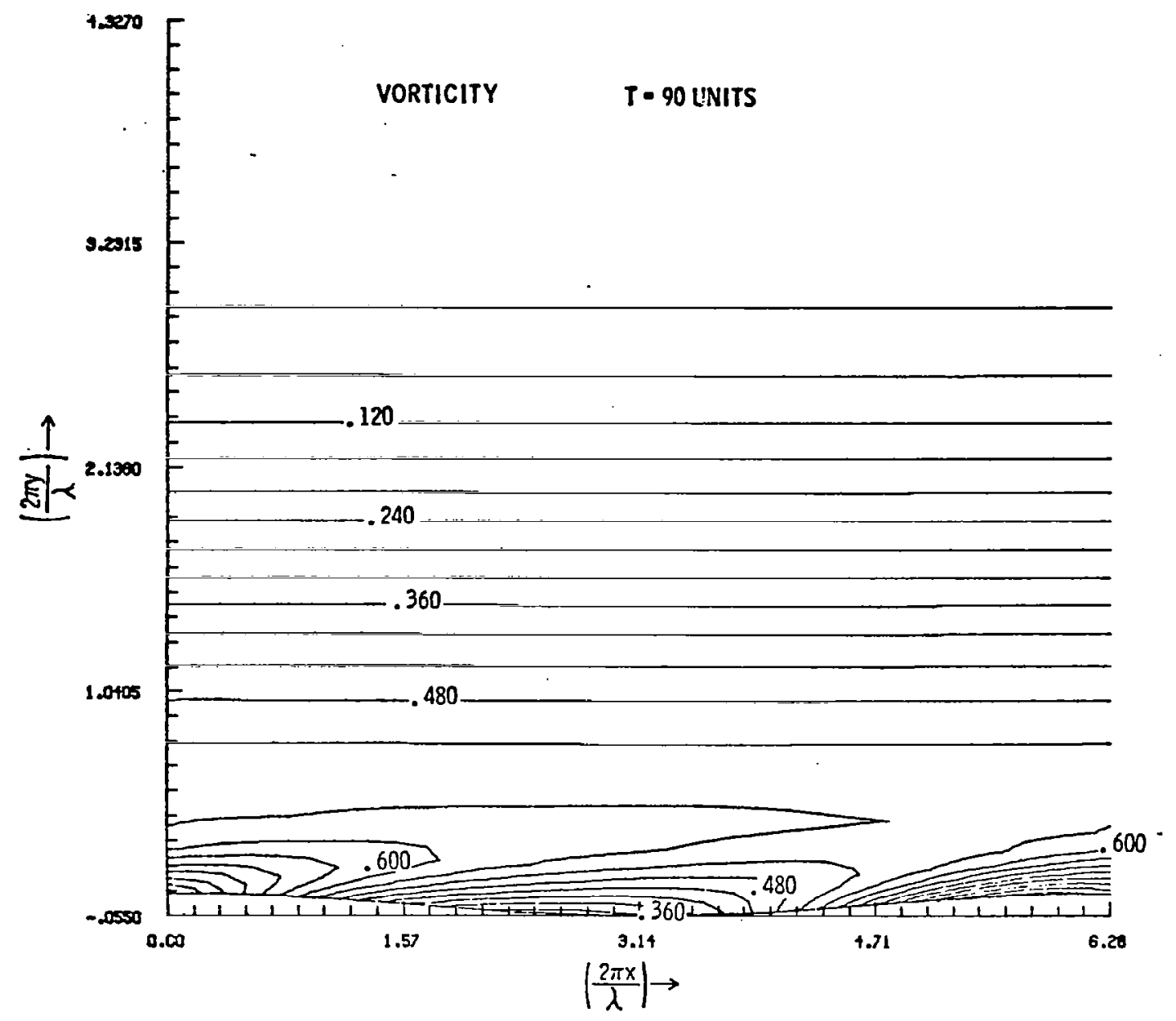

Figure 11. Contour plot of the spanwise vorticity in Run 2 at $t=90$. 


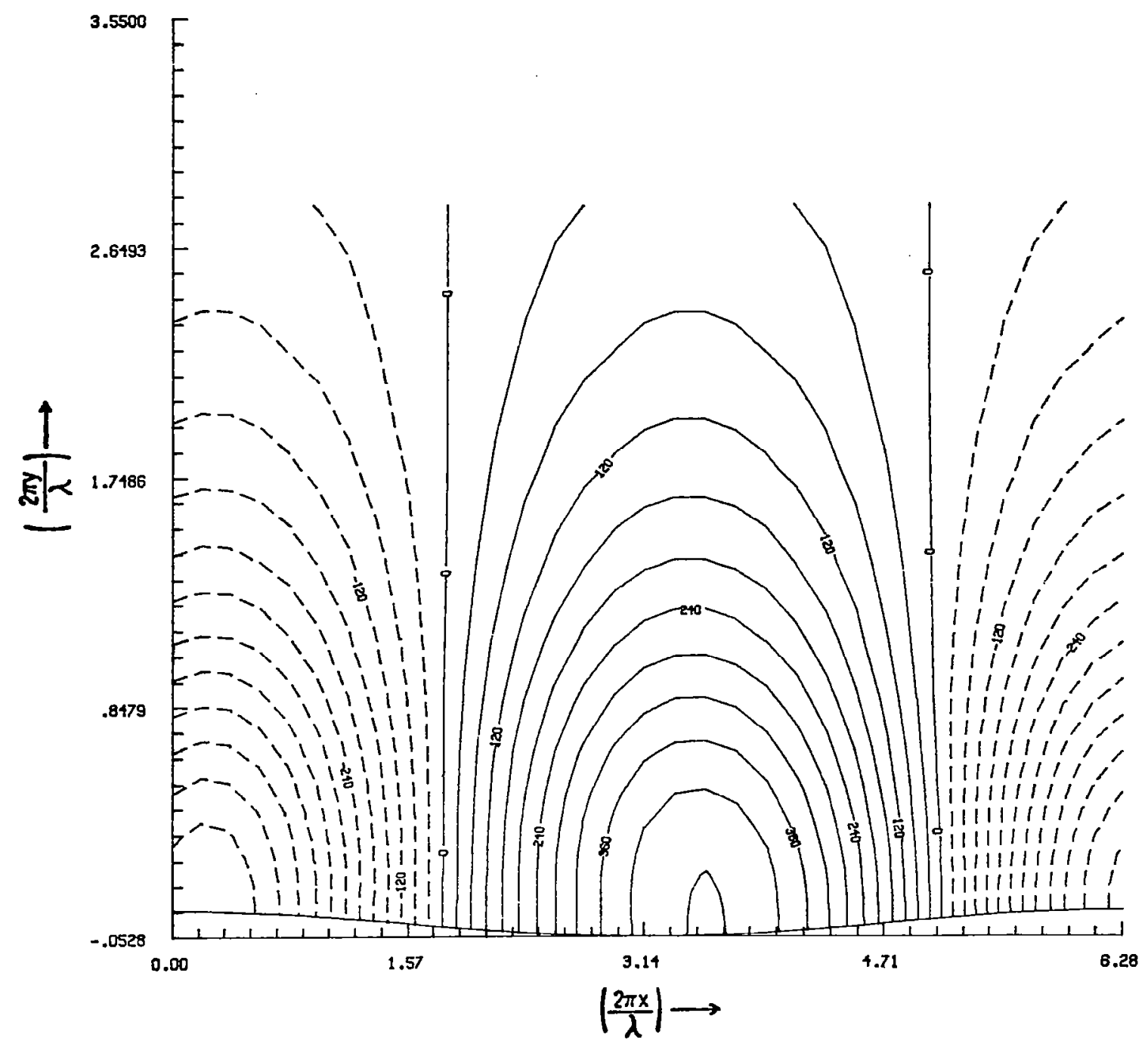

Figure 12. Contour plot of the pressure p after a steady state has been achieved in Run 3 . 


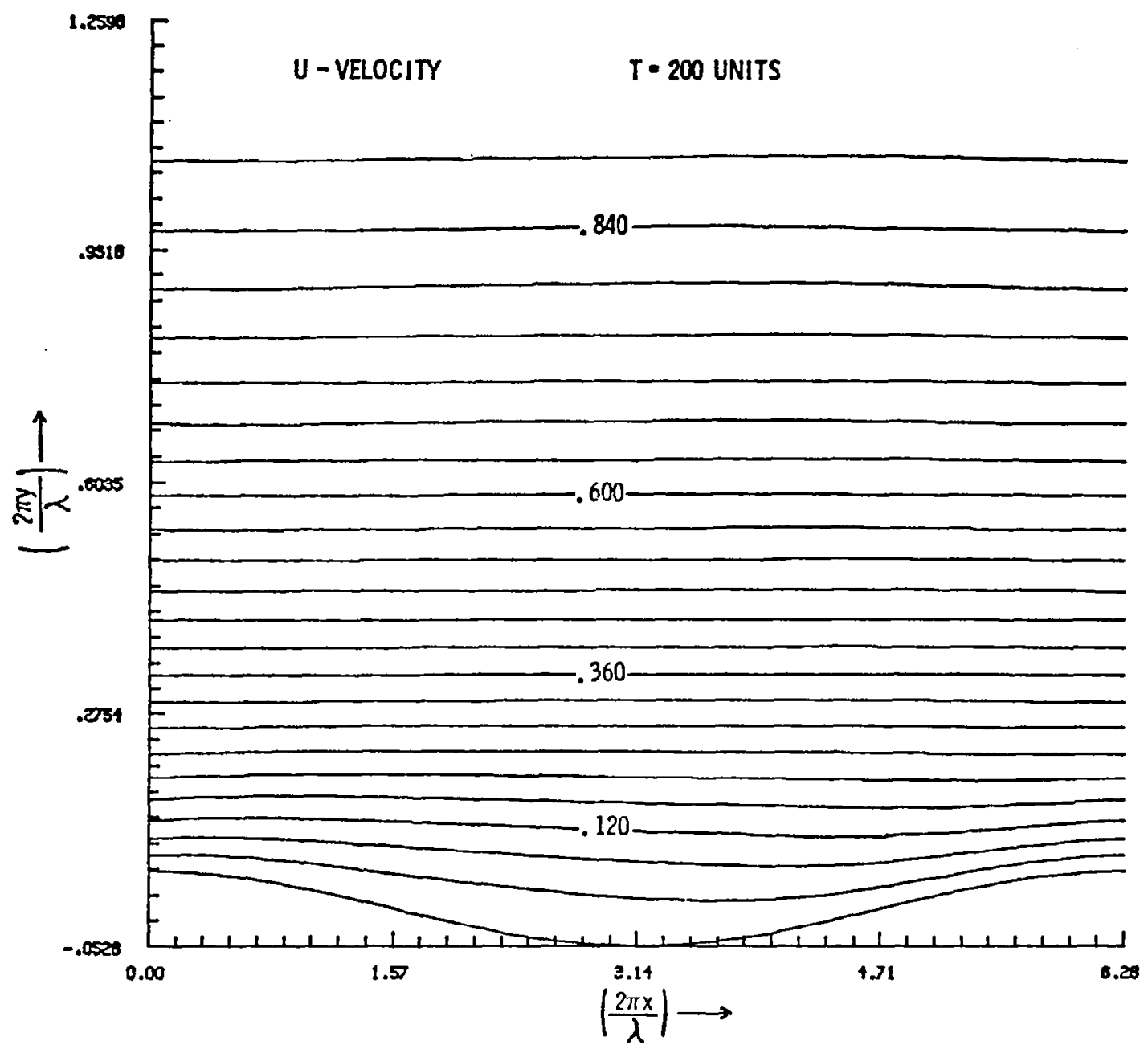

Figure 13. Contour plot of the $x$-velocity $v_{1}$ in Run 3 after a steady state is achieved. 


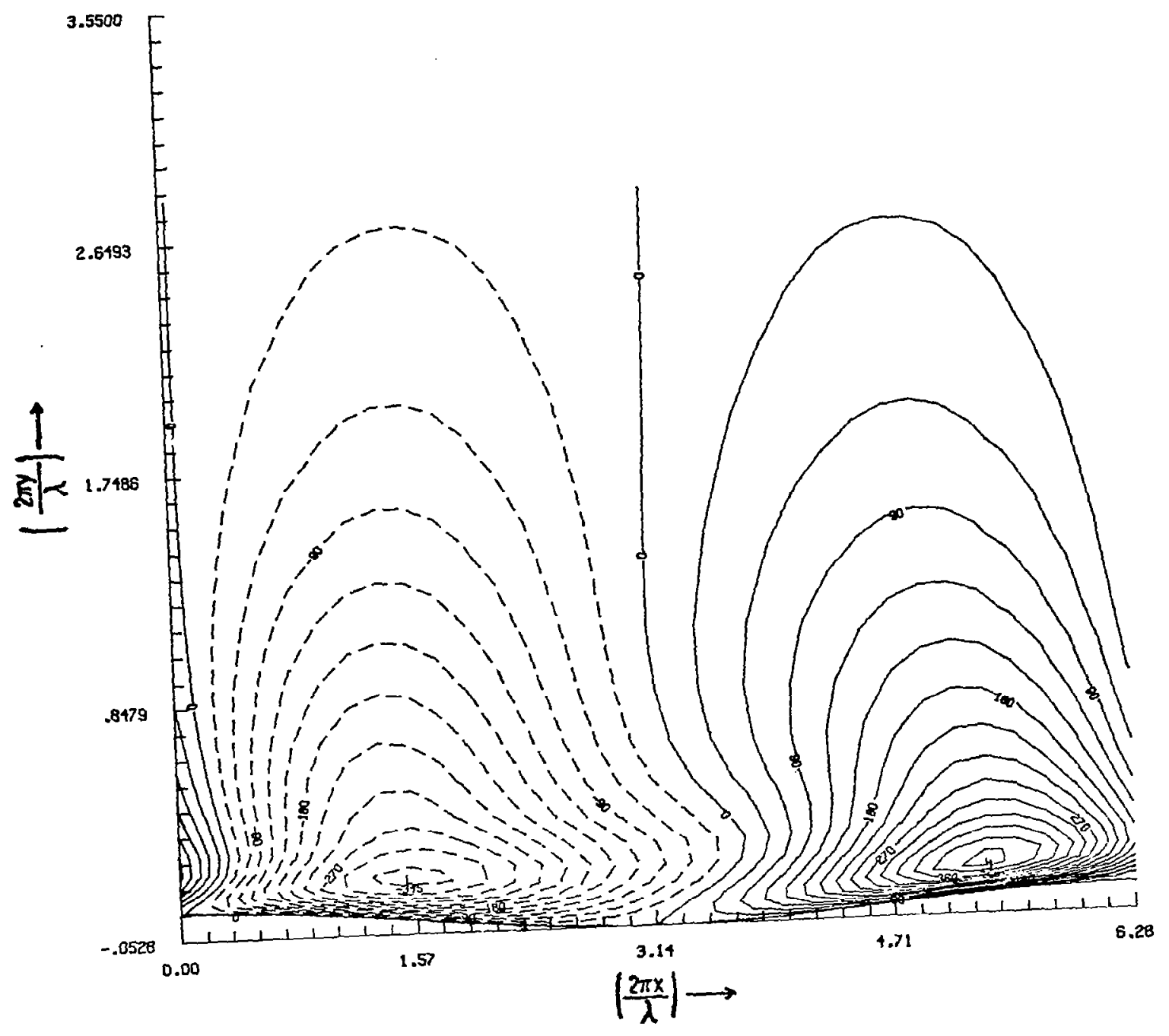

Figure 14. Contour plot of the $y$-velocity $v_{2}$ in Run 3 after a steady state is achieved. 


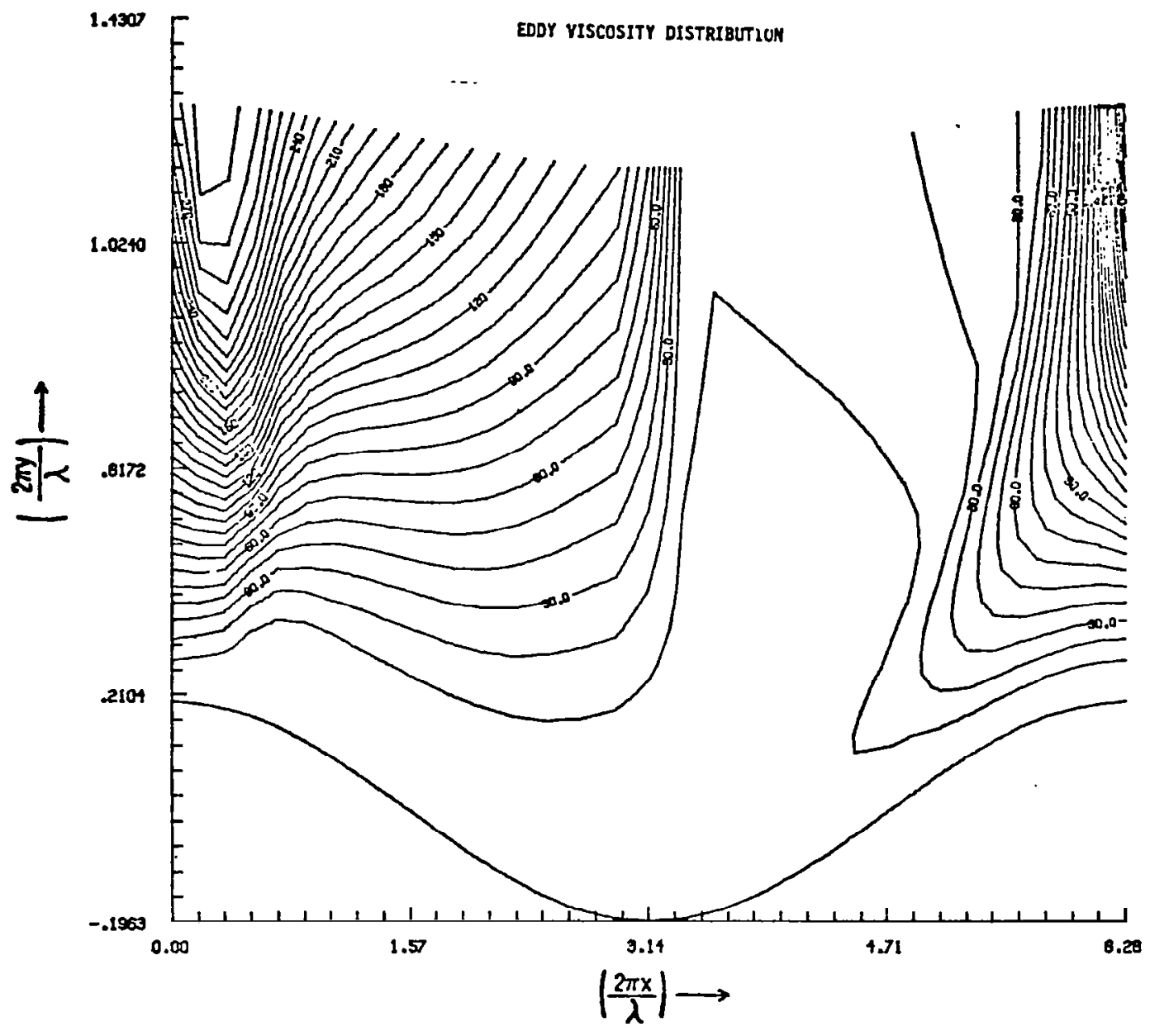

Figure 15. Contour plot of the eddy viscosity coefficient $\mu_{T}$ used to simulate Kendall's experiment. ${ }^{10}$ 


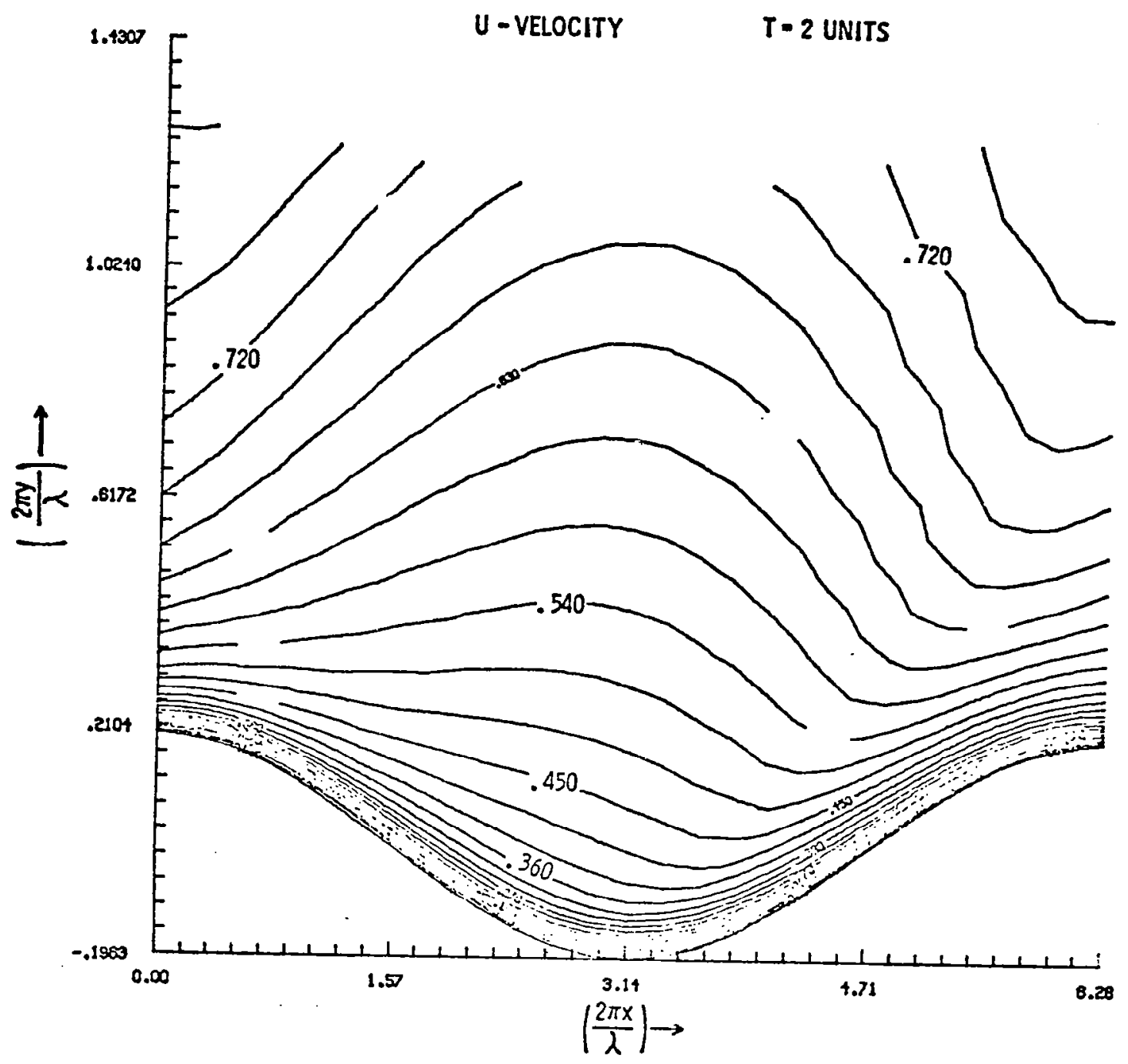

Figure 16. Contour plot of $v_{1}$ at $t=2 s$ in the simulation of Kendall's experiment. 


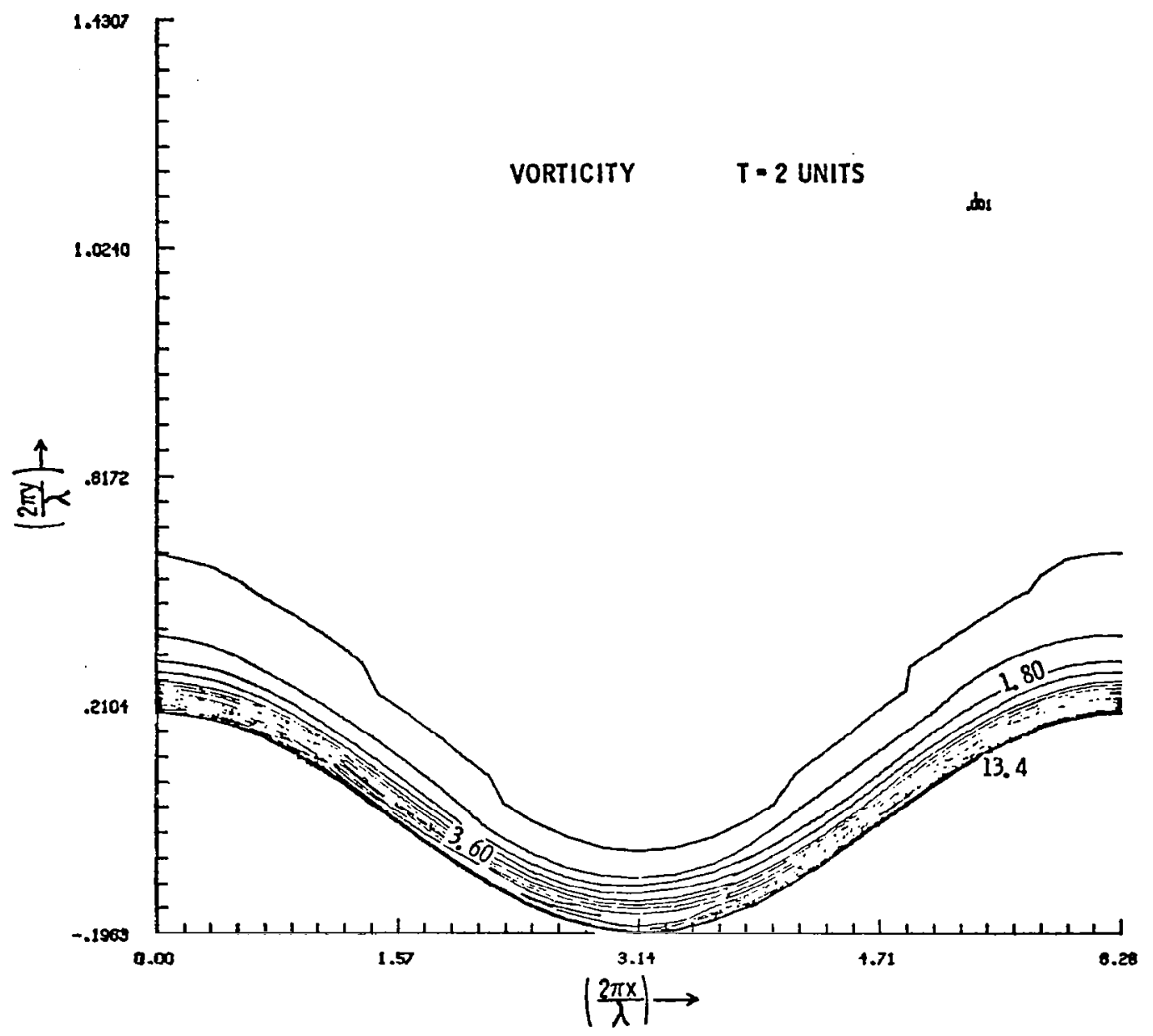

Figure 17. Same as Figure 16 except for the spanwise vorticity. 


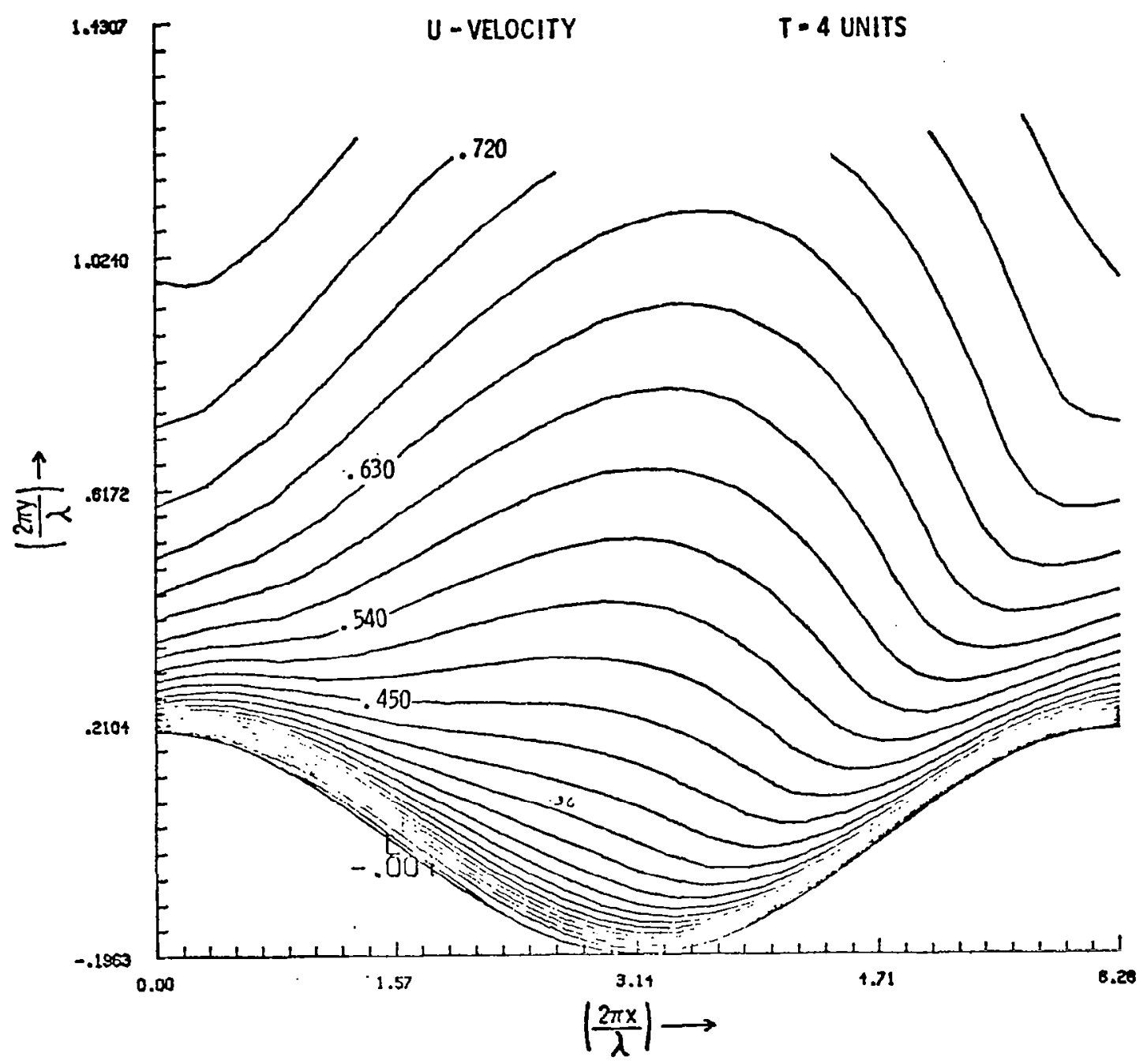

Figure 18. Contour plot of $v_{1}$ at $t=4 \mathrm{~s}$ in the simulation of Kendall's experiment. 


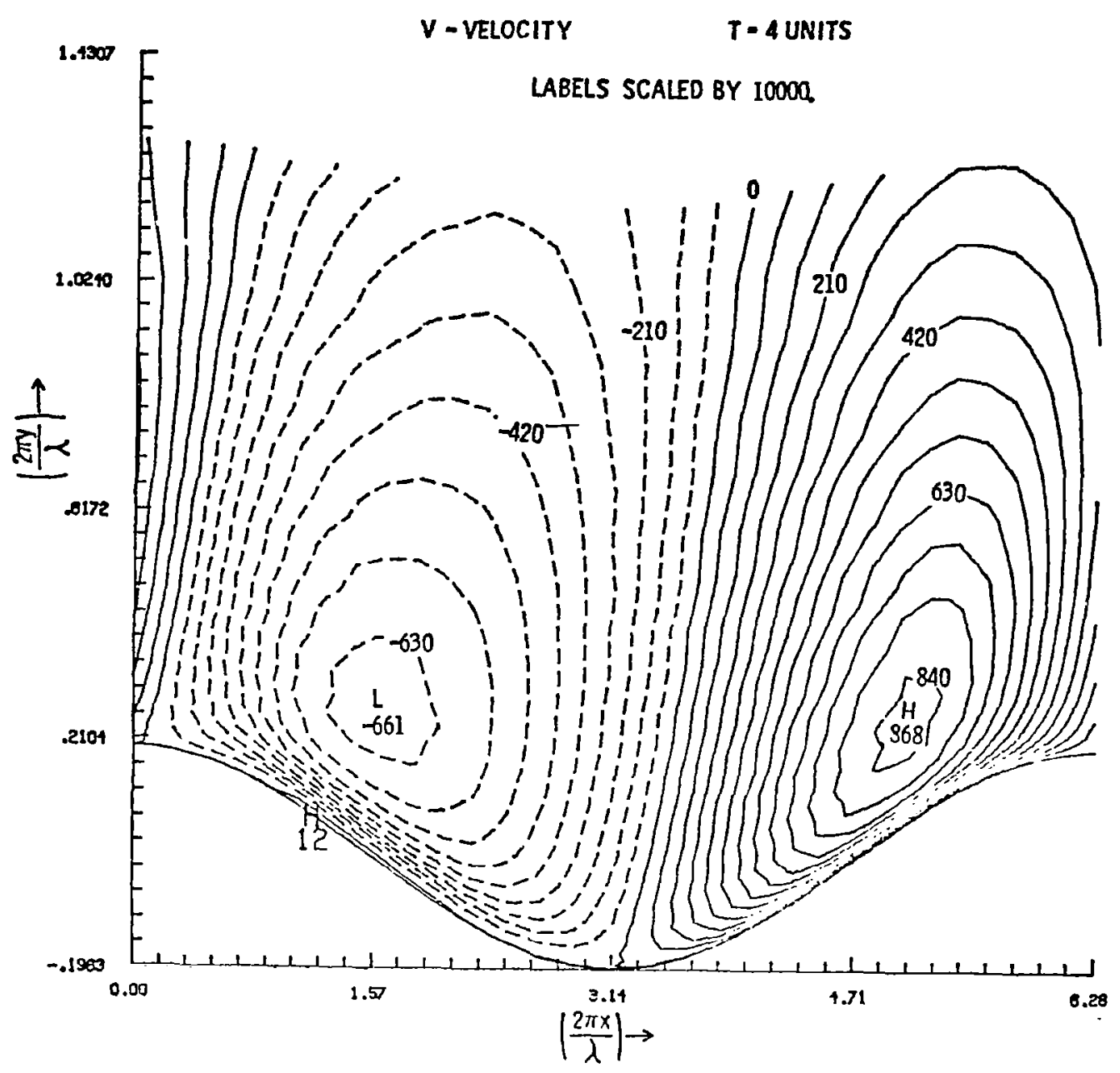

Figure 19. Same as Figure 18 except for $v_{2}$. 


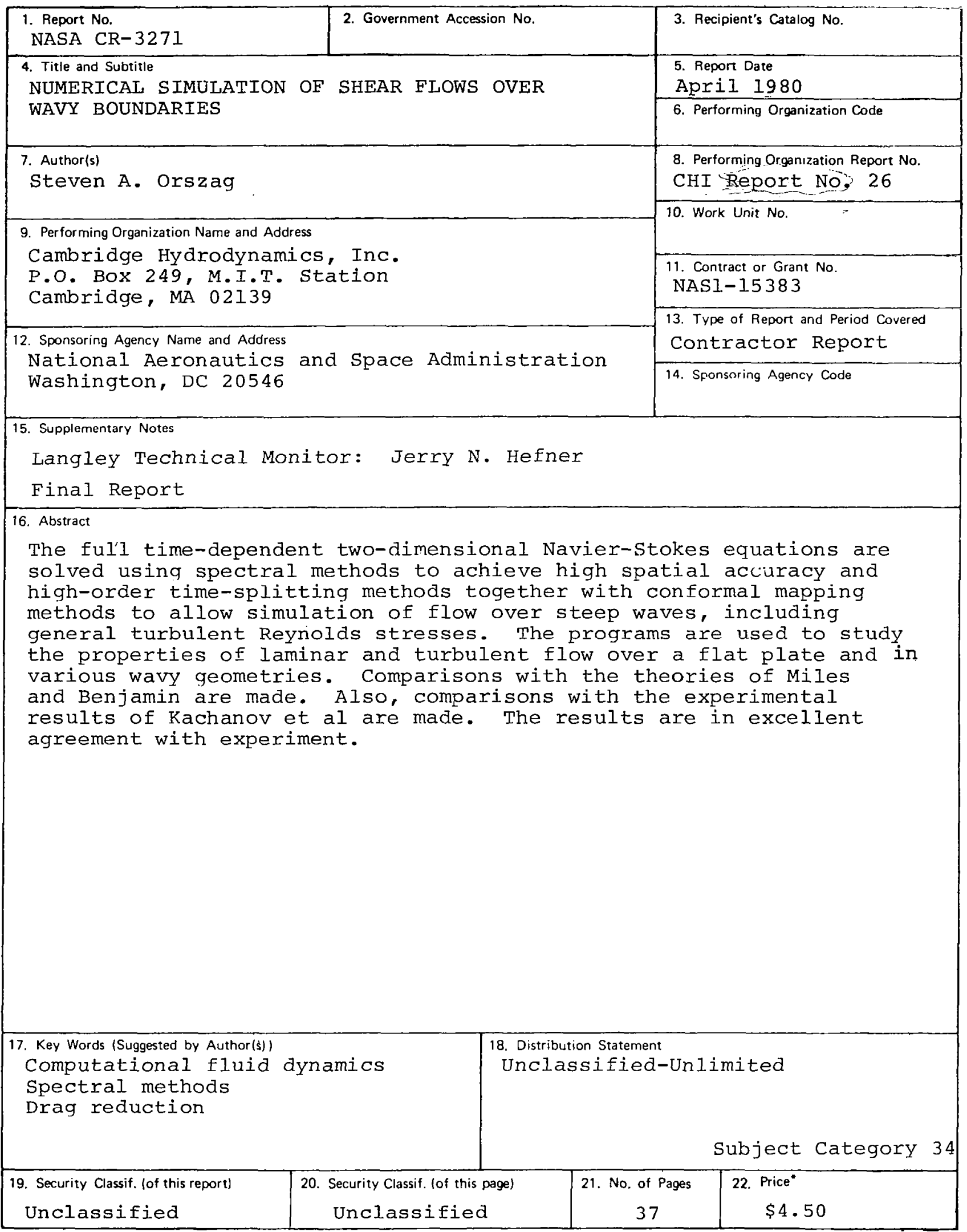

*For sale by the National Technical Information Service, Springfield. Virgınia 22161 